\title{
Multiparameter sensor based on a chaotic fiber-ring resonator
}

\author{
Cesar Jáuregui \\ Optoelectronics Research Centre, University of Southampton, Highfield Campus, SO171BJ, England
}

José M. López-Higuera, Adolfo Cobo, and Olga M. Conde

Photonics Engineering Group, Technología Electrónica, Ingeniería de Sistemas y Automática, University of Cantabria, Avda. los Castros s/n, Santander, 39005, Spain

Joseba Zubía

Plastic Optical Fiber Research Team, Departamento de Electrónica y Telecommunicaciones, School of Engineering, University of the Basque Country, Alda. Urquijo s/n, Bilbao, 48013, Spain

\begin{abstract}
Received March 31, 2006; revised June 26, 2006; accepted June 28, 2006; posted July 6, 2006 (Doc. ID 69475)
A new kind of fiber-optic sensor, to our knowledge, is proposed: a chaotic sensor. The sensor is based on a fiberring resonator that is optically driven by a train of pulses whose period equals the round-trip delay of the resonator. This sensor exploits the extremely high dependence of chaotic systems on initial conditions to sense several physical parameters (elongation, attenuation, index of refraction). The measured information is encoded in different geometrical characteristics of the chaotic attractor. The proposed sensing scheme has been modeled and simulated. The simulations reveal high potential for this structure as a multiparameter sensor. (C) 2006 Optical Society of America

OCIS codes: $060.2370,060.4370$.
\end{abstract}

\section{INTRODUCTION}

One of the most famous characteristics of $\operatorname{chaos}^{1}$ is what is known as the butterfly effect or, in technical language, sensitive dependence on initial conditions. This characteristic accounts for the unpredictability of chaotic systems. It states that extremely small changes in the initial conditions of the system can result in enormous variations of the output. This can be regarded as a problem in most fields but not in sensing. Actually, this premise is good for a sensor, since it speaks of a high sensitivity. In spite of that, little work has been carried out using chaos for sensing purposes. ${ }^{2}$

This paper proposes the use of a chaotic fiber-ring resonator as a sensor for measuring several physical parameters. Among all the possible chaotic systems, the fiberring resonator ${ }^{3}$ is one of the simplest and of highest potential for sensing. The interest in this structure comes from its inherent sensitivity to various physical parameters (index of refraction, fiber length, attenuation, etc.) and, partly, from its simplicity. The fact that it is a simple structure allows for the development of a simplified mathematical model that can be used to retrieve the measured information from the chaotic output of the system. This model is presented and discussed in this paper.

The paper is structured as follows. In Section 2 the principle of operation of the chaotic sensor is discussed. Section 3 is devoted to the mathematical model of the chaotic system. Section 4 deals with the presentation and discussion of multiple simulation results that illustrate the potentiality of this structure as a sensor. Finally, some conclusions are extracted and summarized in the last section.

\section{OPERATING PRINCIPLE}

Chaos is unpredictable, but unpredictability should not be mistaken for randomness. The output of chaotic systems is not random, but it is governed by deterministic mathematical equations. In spite of this, its output cannot be predicted in advance. However, there are certain characteristics that can be foreknown. For example, it is well known that the output will be bound to a certain region of the phase space (that relates the imaginary and real parts of the electric field in our case). This region is known as the chaotic attractor, ${ }^{4}$ or simply attractor. It cannot be predicted what the value of the output in two consecutive observations will be, but it can be affirmed that they will lay within the spatial region defined by the chaotic attractor.

The chaotic attractor is an important feature of a chaotic system. It is like its fingerprint. Moreover, everything that affects the system is reflected in its chaotic attractor in one way or another. Therefore it will be the key parameter for using chaotic systems as sensors. Thus the measured information will be encoded in some geometrical characteristic of the attractor. In following sections we will study the way that different physical parameters alter the attractor of the fiber-ring resonator. 
As can be seen in Fig. 1, the proposed sensor is a conventional fiber ring closed by a fiber-fused biconical $2 \times 2$ coupler. This structure is driven by a pulsed input signal $\left(E_{\text {in }}\right)$ with a period $(\tau)$ that equals the round-trip delay of the light in the fiber ring (of length $L$ ). This is one of the few strong requirements of the proposed sensor. Given this configuration and the proper combination of coupling ratio $(\kappa)$, insertion losses of the coupler $(\rho)$, and input power, a chaotic output $\left(E_{\text {out }}\right)$ is obtained. This chaotic signal is also a train of pulses of period $\tau$, but the amplitudes of the pulses are different and vary in a chaotic manner. Implicitly assuming that there is no intrapulse

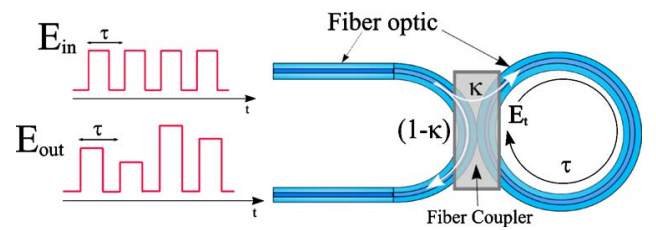

Fig. 1. (Color online) Chaotic fiber-ring resonator driven by a pulsed input signal whose period $(\tau)$ equals the round-trip delay of the fiber ring. variation of the amplitude, this signal can be treated as discrete even though it is continuous in time. The same is valid for the input signal.

Figure 2 shows what is known as bifurcation diagram of the proposed sensor (for a fiber ring of $1000 \mathrm{~m}$ length). This kind of diagram shows the evolution of the output as a function of the input power. At each input power, all the possible values of the amplitude of the output pulses are represented. It reveals the four different operating states of a chaotic system: stable, periodic, quasiperiodic, and chaotic. The first of these operating states is characterized by a constant output amplitude [inset (a) of Fig. 2]. The second state corresponds to a periodic output that will force the output to vary between different discrete values [insets (b) and (c) of Fig. 2]. On the other hand, the quasiperiodic state is characterized by an output that resembles a periodic signal but without a perfect periodicity. Finally, the chaotic state [inset (d) of Fig. 2], the one of interest for this work, is characterized by a signal whose amplitude varies in an unpredictable way.

One of the most interesting features of bifurcation diagrams is that they reveal the evolution from one operat-
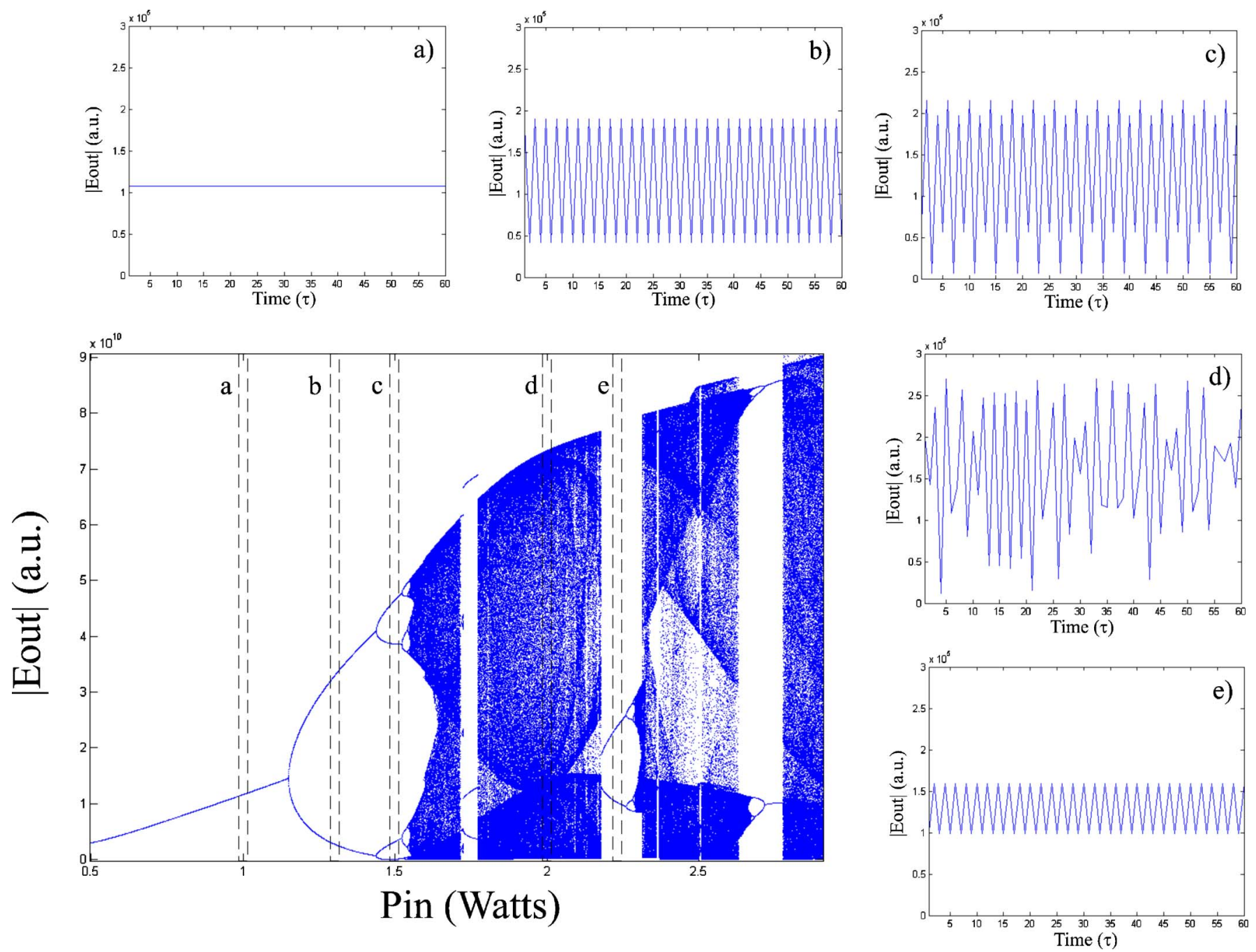

Fig. 2. (Color online) Bifurcation diagram of the proposed chaotic sensor. This diagram shows the different possible working states of the system as a function of the input power: (a) stable state, (b) periodic state (two values), (c) periodic state (four values), (d) chaotic state, (e) periodic state (two values). 


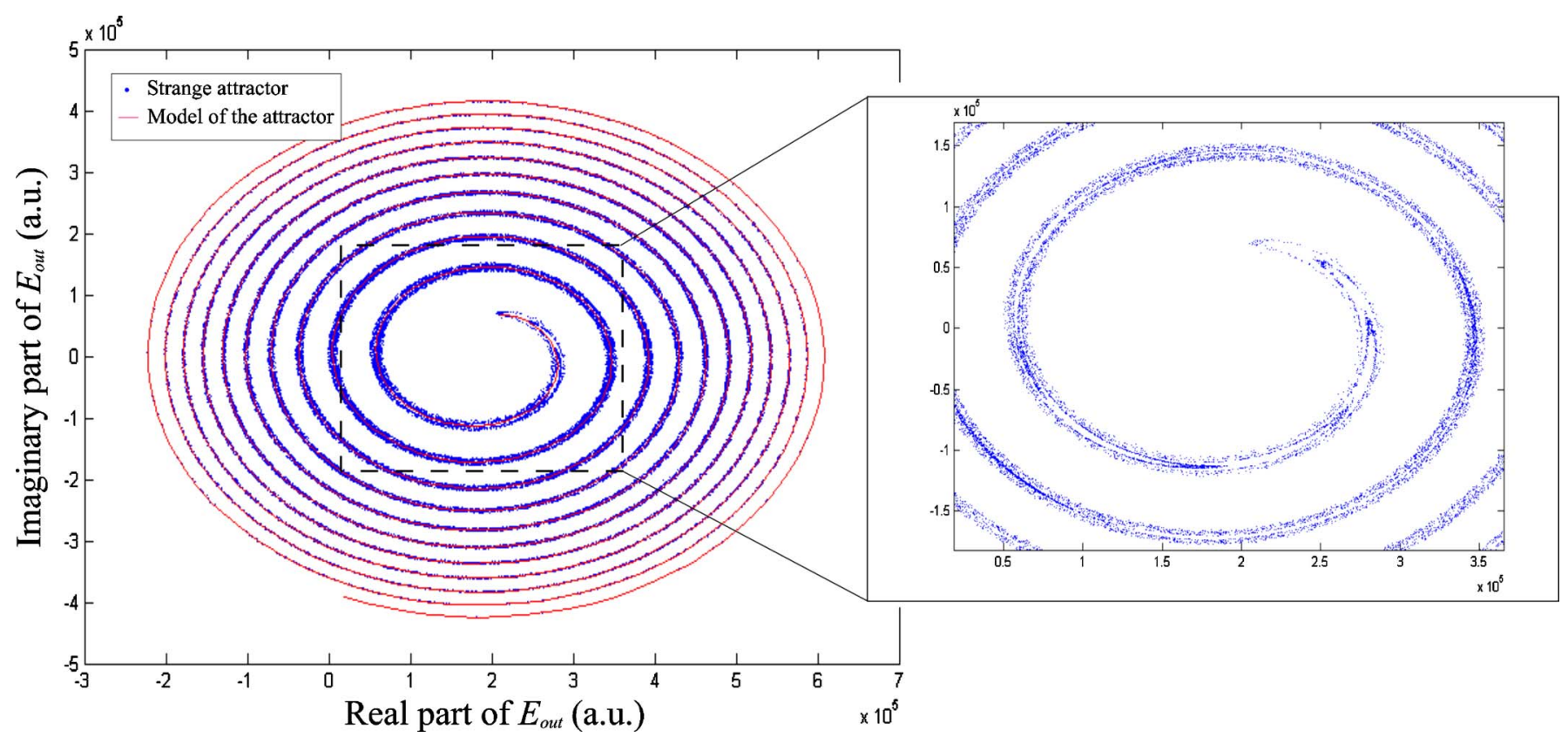

Fig. 3. (Color online) Chaotic attractor of the proposed fiber-ring chaotic sensor. The fit provided by the mathematical model presented in the paper is also shown. The inset shows a close-up of the central part of the attractor.

ing state to another. Thus, it can be seen that for small input powers the output of the system is a train of pulses of constant amplitude. However, there is a point, around $1.2 \mathrm{~W}$ input power, at which the diagram bifurcates into two values, giving rise to a periodic output signal. Around $1.45 \mathrm{~W}$ the diagram bifurcates once more, generating a four-value periodic output signal. From that point on, the bifurcations of the diagram take place closer and closer, creating what is called the bifurcation route to chaos. At the end of this process there are so many possible values for the amplitude of the output signal that no periodicity can be found anymore. This is the chaotic regime and is represented by the dark bands of the bifurcation diagram. However, in contrast to what might be thought at first, a high input power alone does not guarantee a chaotic state. The bifurcation diagram shows that the chaotic regimes are interrupted by periodic operating states [for example, inset (e) of Fig. 2].

In the chaotic regime, the chaotic attractor of the sensor is revealed by our representing the output electric field $\left(E_{\text {out }}\right)$ in the phase space. As shown in Fig. 3, it takes the gross form of a spiral. However, a zoom (see the inset of Fig. 3) soon reveals a much more complex inner structure. The spiral is formed by lines that fold themselves but never cross one another. The existence of this complex inner structure makes it impossible to describe the actual shape of the attractor with an algebraic expression. However, its gross form is simple enough to be modeled. This model is described in Section 3.

\section{MATHEMATICAL MODEL OF THE CHAOTIC ATTRACTOR}

Since the chaotic attractor is the feature of the system sensitive to external parameters, a mathematical model that reveals these dependencies is required. Thus, the sensing procedure will be as follows: First of all, enough output pulses to obtain a well-defined attractor will be recorded; these output pulses (electrical field) will be represented in the phase space to draw the attractor; finally the attractor will be fitted using the mathematical model that is presented below; from this fit the measurands will be recovered. This way, importance of developing an accurate parametric mathematical model (whose parameters are the physical magnitudes to sense) becomes clear.

Before presenting the model, we need to establish the nomenclature and make some assumptions: the fiber-ring resonator is formed by a standard telecommunication fiber of length $L \mathrm{~m}$; the fiber presents an attenuation factor of $\alpha \mathrm{Np} / \mathrm{m}$; and the fiber ring is closed with a coupler whose coupling coefficient is $\kappa$ and that has a linear insertion loss coefficient of $\rho$. Finally, as can be seen in Fig. 1, the resonator is driven by a pulsed input signal $E_{\text {in }}$ of periodicity $\tau$ that matches the round-trip delay of the ring (i.e., $\tau=L n_{1} / c$, where $c$ is the speed of light in vacuum).

The starting point for developing the model is to notice that all chaotic systems are nonlinear, i.e., demand underlying nonlinear processes. ${ }^{4}$ In this particular case the nonlinear medium is the optical fiber that forms the fiber ring. Fibers have intrinsic nonlinearities in their index of refraction that can be expressed by

$$
n(z)=n_{1}(z)+n_{2}|\widetilde{E}(z)|^{2}+\cdots .
$$

In this equation $\widetilde{E}(z)$ stands for the electric field inside the fiber, $n_{1}(z)$ is the linear index of refraction of the fiber, $n_{2}(z)$ is the quadratic index of refraction, and so on. As can be seen, their spatial dependence has been explicitly considered to account for possible inhomogeneous sections or for local external perturbations. However, for simplicity, the spatial dependency will be dropped from the notation, although it was considered when developing the model. Moreover, as usual with fibers, only the indices up to quadratic order will be considered. 
Taking into account Eq. (1), it can be shown that in a fiber ring the electric field at the input point $\left(E_{t}\right)$ is given by the following recursive equation:

$$
\begin{aligned}
E_{t}(t)= & j \sqrt{1-\rho} \sqrt{\kappa} E_{\mathrm{in}}(t)+\sqrt{1-\rho} \sqrt{1-\kappa} E_{t}(t-\tau) \\
& \times \exp \left(-\frac{\alpha L}{2}\right) \exp \left(-j \frac{2 \pi}{\lambda}\left\{n_{1} L+n_{2}\left|E_{t}(t-\tau)\right|^{2}\right.\right. \\
& \left.\left.\times\left[\frac{1-\exp (-\alpha L)}{\alpha}\right]\right\}\right) .
\end{aligned}
$$

And, on the basis of Eq. (2), the output electric field $E_{\text {out }}$ is

$$
\begin{aligned}
E_{\text {out }}(t)= & \sqrt{1-\rho} \sqrt{1-\kappa} E_{\text {in }}(t)+\frac{j \sqrt{\kappa}}{\sqrt{1-\kappa}}\left[E_{t}(t)\right. \\
& \left.-j \sqrt{1-\rho} \sqrt{\kappa} E_{\text {in }}(t)\right] .
\end{aligned}
$$

Both equations present the time variable $t$ as a continuous magnitude. However, for the reasons already explained, it will be treated as discrete: time will change in integer multiples of $\tau$. Besides, even though not explicitly noted, the input electric field $E_{\text {in }}$ incorporates noise. Thus, this electric field can be expressed as the sum of two terms: $E_{\text {in }}(t)=\widetilde{E}_{\text {in }}(t)+n_{\text {in }}(t)$, where $\widetilde{E}_{\text {in }}(t)$ is a constantamplitude input electric field and $n_{\text {in }}$ is noise. Owing to the sensitive dependence of chaotic systems on initial conditions, the incorporation of the noise term in the model is mandatory.

With Eqs. (2) and (3), the output of the chaotic resonator can be simulated, and graphs similar to those in Fig. 2 can be obtained. The model gives a complex output electric field that, when plotted in the phase space, generates the attractor. Then the attractor can be parametrized using this model to carry out simulations. By this procedure it can be demonstrated, for example, that the center of the spiral is given by $\sqrt{1-\rho} \sqrt{1-\kappa}\left|E_{\text {in }}\right|$.
One of the main parameters of any spiral is the evolution of its radius $\left(r_{s}\right)$ with each turn. For the case of the attractor of the sensor proposed in this paper the evolution of the radius is always similar to that shown in Fig. 4. This kind of evolution can be described by a Belehdarek function: $r_{s}=r \sqrt{\phi / \pi}$, where $\phi$ is the number of turns expressed in radians. This fit has a parameter $r$ that can be regarded as the radius evolution rate. Thanks to the simulations, it can be seen that this parameter mainly depends on the wavelength of the light $(\lambda)$ and on the length of the fiber ring $(L)$. Thus, the dependence of $r$ on the ratio $L / \lambda$ is shown in Fig. 5. A parametric expression for the radius of the spiral can be derived from that graph:

$$
r_{s}=r \sqrt{\frac{\phi-\phi_{o}}{\pi}}, \quad \text { with } r=a\left(\frac{L}{\lambda}\right)^{\beta} \text {, }
$$

where $a$ and $\beta$ are fitting parameters of the model and $\phi_{o}$ is an angular offset that accounts for different starting radii. The explicit dependence of this radius change rate with the length $L$ of the cavity ring will be exploited in Section 4 for sensing strain.

Parameters $\alpha$ and $\beta$ of Eq. (4) can also be expressed as a function of the parameters of the fiber ring. Finally, a closed and fully parametric expression for the whole spiral is obtained:

$$
\begin{array}{r}
A t=\sqrt{1-\kappa} \sqrt{1-\rho}\left|E_{\mathrm{in}}\right|+F_{\rho} F_{\kappa} a\left(\frac{L}{\lambda}\right)^{\beta} \sqrt{\frac{\phi-\phi_{o}}{\pi}} \exp (-j \phi), \\
\phi_{i} \leq \phi \leq \phi_{f}, \quad(5)
\end{array}
$$

with

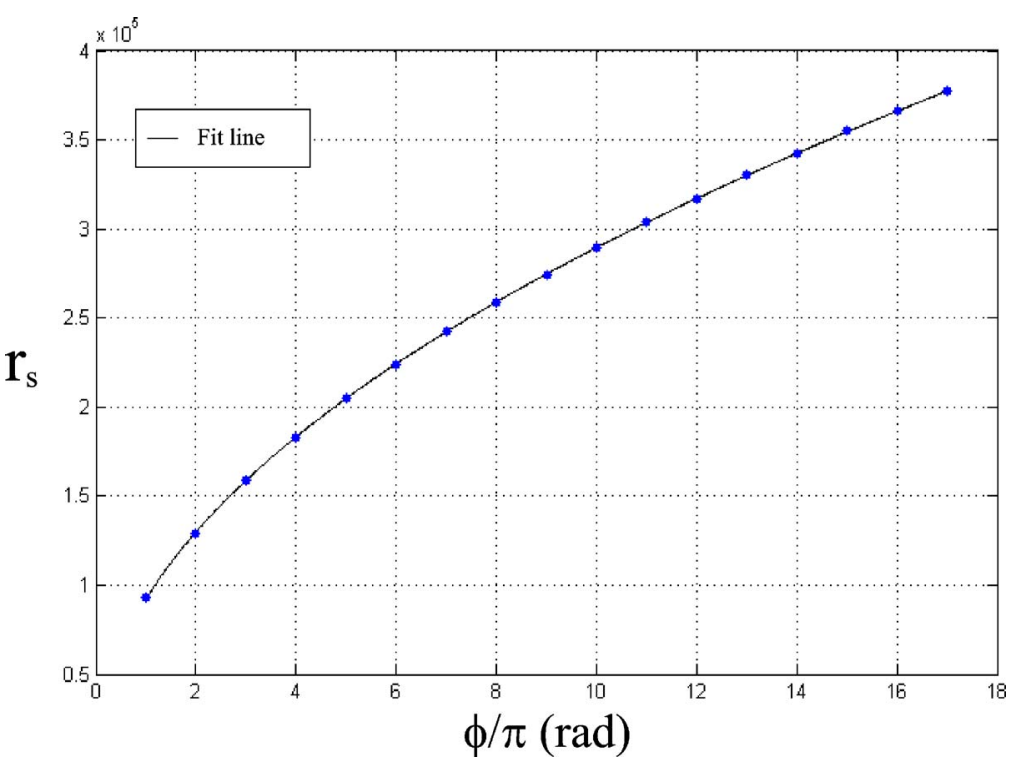

Fig. 4. (Color online) Evolution of the radius of the spiral with each half-turn (dotted curve) together with the fit provided by the Belehdarek fit (solid curve). 


$$
\begin{gathered}
\beta=-0.12236 \alpha-0.49527, \\
F_{\rho}=-0.21113 \rho^{2}-0.5928 \rho+1.1961, \\
F_{\kappa}=-0.2304 \kappa^{2}+1.0188 \kappa+0.36663, \\
a=p_{1} n_{1}+p_{2}, \\
p_{1}=-2.8646 \times 10^{3}\left(\alpha+3.6555 \times 10^{4}\right), \\
p_{2}=-4.9471 \times 10^{3}\left(\alpha+5.3399 \times 10^{4}\right) .
\end{gathered}
$$

In Eqs. (6) and (7) the attenuation of the fiber $(\alpha)$ is expressed in decibels per kilometer. This model is valid only in a certain region given by $1.4 \leq n_{1} \leq 1.5,0.1 \leq \alpha$ $\leq 1.5 \mathrm{~dB} / \mathrm{km}, 0.45<\kappa \leq 0.99$, and $001 \leq \rho \leq 0.5$. The model does not posses fundamental limits on the fiber length; however, there are some practical constraints. For example, to obtain accurate results the spiral of the attractor should have, at least, one complete turn. On the other hand, for long lengths of fiber, many pulses are required

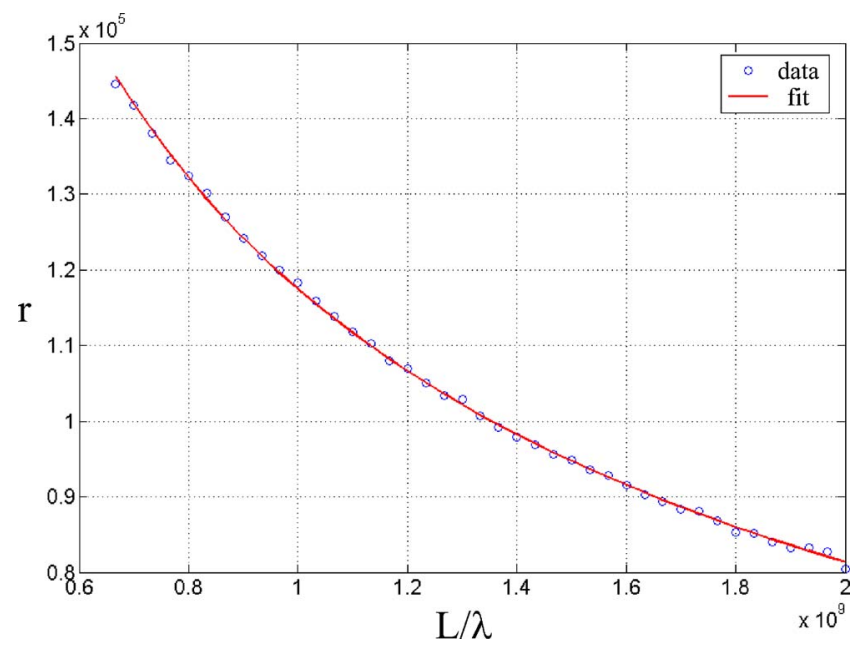

Fig. 5. (Color online) Dependence of the radius evolution rate on the $L / \lambda$ ratio (dotted curve) and its fit revealing a potential relationship (solid curve).

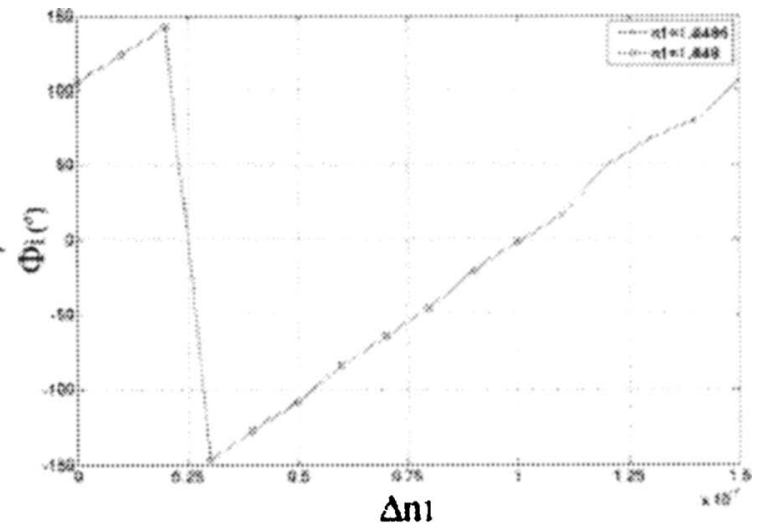

Fig. 6. Behavior of the initial angle of the attractor $\left(\phi_{i}\right)$ with length (left graph) and refractive index change $\left(\Delta n_{1}\right)($ right graph). The left graph also shows how the dependence of $\phi_{i}$ on the fiber length varies with the index of refraction $n_{1}$. The right graph shows the evolution to define the outer regions of the attractor. Thus, a practical length range for the sensor could be $\sim 900 \leq L$ $\leq 3000 \mathrm{~m}$.

Apparently, the model does not exhibit any dependence with $n_{2}$. This does not mean that the attractor does not depend on this parameter. It means that the model has been derived just for standard telecommunication fibers that usually have similar nonlinear indices of refraction. Therefore, in the model this parameter has been left constant with a value of $8 \times 10^{-14} \mathrm{esu}$.

On the other hand, it can be seen in Eq. (5) that the model depends on three angles: $\phi_{i}, \phi_{f}$, and $\phi_{o}$. The first two represent the initial and final points of the attractor, respectively, and the third one provides the angular offset required to best fit the actual attractor. Therefore these parameters are important to completely describe the attractor. Moreover, they will reveal themselves essential to perform measurements of the refractive index of the fiber.

These parameters also depend on the physical configuration of the chaotic fiber-ring resonator. Figure 6 shows, on its left-hand side, the dependence of the initial angle of the attractor $\left(\phi_{i}\right)$ on the length of the fiber for different indices of refraction. The most significant characteristic of this graph is that this relationship between $\phi_{i}$ and length is always linear (this also holds for $\phi_{f}$ and $\phi_{o}$ ). Moreover, as clearly shown in the right-hand side of Fig. 6, the slope of these straight lines varies smoothly with the index of refraction. This graph shows the evolution of $\phi_{i}$ for a $1500 \mathrm{~m}$ long ring when the index of refraction of the fiber changes. It shows a periodic sawtooth variation with period of $15 \times 10^{-8}$. This period will ultimately determine the dynamic range of the sensor when index changes are measured. On the other hand, this periodicity is exactly the same no matter what the original refractive index of the fiber is. This is demonstrated in the graph by superposing two evolutions of $\phi_{i}$ corresponding to two initial indices of refraction: 1.448 (dotted curve) and 1.4486 (crossed curve). Thus, from the data obtained from Fig. 6, and in the linear region of the sawtooth signal, one can establish the following mathematical model for these angular parameters:

$$
\phi_{v}=\left(a a_{\phi_{v}} n_{1}+b a_{\phi_{v}}\right) L+\left(a b_{\phi_{v}} n_{1}+b b_{\phi_{v}}\right) \text {, with } v=i, f \text {, or } o \text {. }
$$

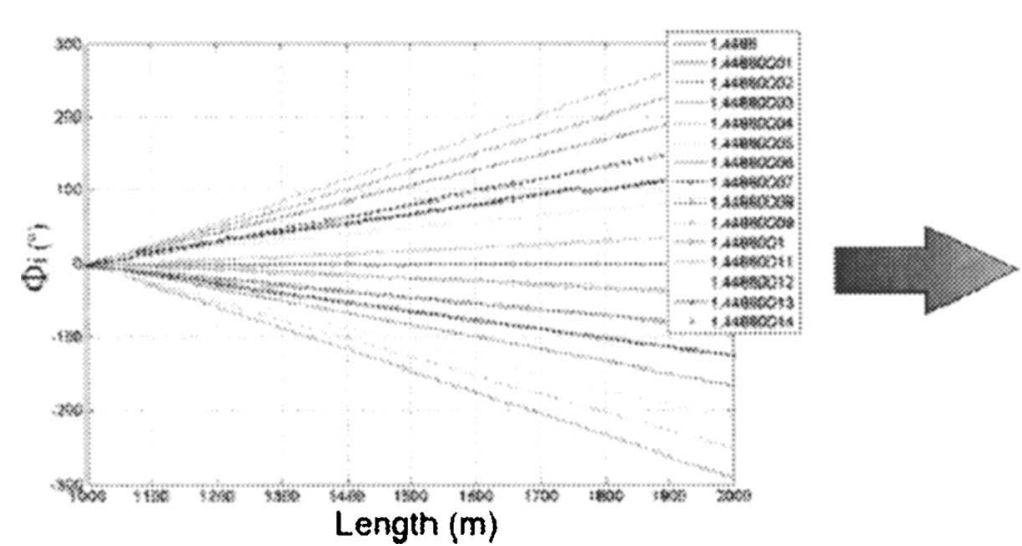

Fig. 6. Behavior of the initial angle of the attractor $\left(\phi_{i}\right)$ with length (left graph) an
graph also shows how the dependence of $\phi_{i}$ on the fiber length varies with the index
of $\phi_{i}$ for two indices of refraction (1.448, dotted curve, and 1.4486, crossed curve). 
It is also to be expected that the coefficients $a a_{\phi_{v}}, b a_{\phi_{v}}$, $a b_{\phi_{v}}$, and $b b_{\phi_{v}}$ depend on the rest of the physical parameters of the fiber ring: $\alpha, \kappa$, and $\rho$. However, these dependencies have not been studied in this work.

Figure 3 provides a means for evaluating the quality of the fit given by this model. This figure shows the chaotic attractor of a $2400 \mathrm{~m}$ long fiber-ring resonator, excited by an input pulse train of $5 \mathrm{~W}$, together with the fit provided by the model for this particular ring configuration. It can be seen that the fit for the gross form of the attractor is good.

After the chaotic attractor is fitted, several parameters will be known: $L, n_{1}$, and $\alpha$ are the most useful of them. This suggests that this structure will be, when properly used, able to detect and to measure a wide range of parameters. For example, this sensor can be used to measure elongation (induced by strain, for example), any parameter altering the index of refraction of the fiber (electrical current, for example), and changes in the attenuation of the fiber ring.

\section{SIMULATION RESULTS}

The main objective of this paper is to propose and demonstrate the feasibility of the chaotic fiber-ring resonator as a sensor. It is, however, out of the scope of this work to propose a suitable interrogation technique for the sensor. It must be said, nevertheless, that there is ongoing work to develop such an interrogation technique. However, since no interrogation technique has been developed yet, only simulation results will be presented and discussed in this section to evaluate the potential performance of the chaotic sensor.

The interrogation of the chaotic sensor will be based on recording as many pulses as possible (limited by the desired interrogation speed) to obtain a well-defined attractor. The recording of the pulses is not a difficult task in itself, since it is not necessary to record every single output pulse. It does not matter if we miss some pulses or record one out of a hundred. The sampling does not even have to be periodic. The fractal nature of the attractor ensures that we will obtain the same result regardless of the temporal characteristics of the sampling.

In the following, simulation results using the proposed structure as a sensor for length, attenuation, and index of refraction will be presented and discussed. In many realworld applications these parameters will vary simultaneously: for example, temperature will modify both the length of the fiber and its index of refraction. However, in most cases the proposed sensor will be able to simultaneously measure two parameters, provided that they are encoded in different geometrical characteristics of the attractor. Thus, in the example above, the sensor will be able to indirectly measure temperature by simultaneously determining the index of refraction and the length of the fiber by solving the system of Eqs. (4) and (8).

Unless otherwise stated, the characteristics of the system will be input optical power of $5 \mathrm{~W}$ launched into a standard telecommunications fiber of $9 \mu \mathrm{m}$ core diameter. The linear refraction index $n_{1}$ is 1.448 , and the secondorder nonlinear index is $8 \times 10^{-14} \mathrm{esu}$. The fiber is consid-

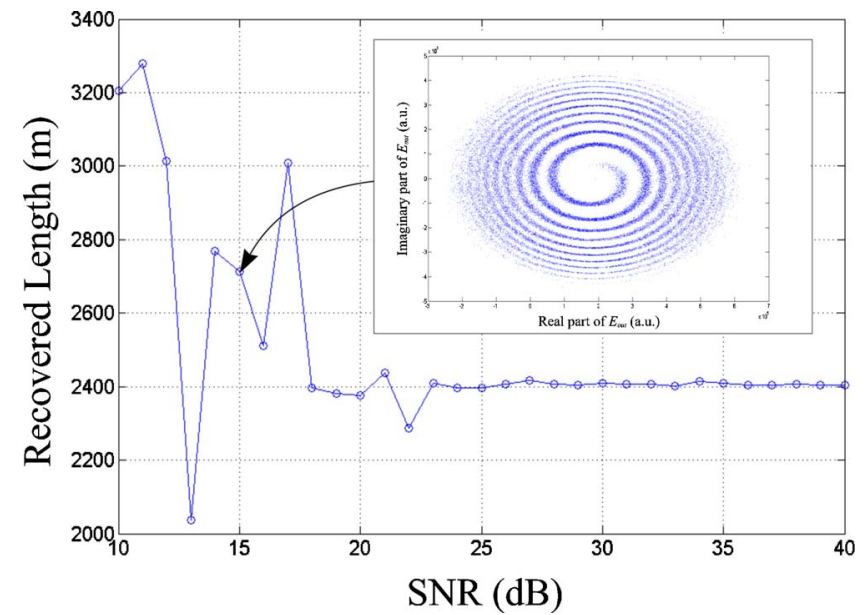

Fig. 7. (Color online) Fiber length as recovered by the proposed sensor when the SNR of the input train of pulses is varied. The inset shows the attractor for a SNR of $15 \mathrm{~dB}$. The noise blurs the spiral.

ered to have an attenuation factor of $0.3 \mathrm{~dB} / \mathrm{km}$. The insertion loss coefficient $\rho$ of the coupler is 0.1 ; meanwhile, its coupling ratio $\kappa$ is 0.75 . The wavelength of the light is $1500 \mathrm{~nm}$. The signal-to-noise ratio (SNR) of the input signal is $40 \mathrm{~dB}$.

As already discussed, the chaotic attractor has a strong dependence with the length of the fiber ring $L$. Figure 5 shows the dependence of the radius change rate of the spiral with the length of the fiber ring when this parameter is swept from 1100 to $3000 \mathrm{~m}$. Therefore, this graph can be used to recover the length of the fiber once the parameter $r$ has been obtained from the chaotic attractor of the sensor. However, in a simpler approach, Eq. (4) can be used instead to recover the length (or even the wavelength of the light if the length of the fiber is known).

Thus, as suggested above Eq. (4) was used to recover the length of a $2400 \mathrm{~m}$ long fiber-ring resonator. In this experiment the SNR of the input signal was swept from 10 to $40 \mathrm{~dB}$. The attractor was formed by 50,000 pulses. The interest of this experiment has to do with the high sensitivity of chaotic systems to initial conditions. Thus, it is worth while trying to determine whether some noise in the input train of pulses seriously affects the output. In the case this happens the whole proposed sensor would be useless. The results of this experiment are shown in Fig. 7. It can be seen that the recovered length is close to the actual value for SNRs of $23 \mathrm{~dB}$ and above. In fact, the average recovered length in this region is around $2404 \mathrm{~m}$, which gives an average error of around $0.2 \%$. In several successive experiments under the same conditions this error was always well below $1 \%$. Therefore, for high enough SNRs, the accuracy of the system in determining the fiber length is fairly good. It was also observed that, for SNRs between 30 and $40 \mathrm{~dB}$, the recovered length in successive experiments at the same SNR exhibited fluctuations of around $\pm 2 \mathrm{~m}$. This means that this sensor is able to detect without ambiguity changes in length of approximately $2 \mathrm{~m}$. This is the same as saying that the sensor has a strain resolution of $\pm 100 \mu \varepsilon$. However, this value can be further improved by recording more output pulses or using a shorter fiber length (since the sensitivity of the sys- 
tem decreases with length, as shown in Fig. 5). For SNRs lower than $20 \mathrm{~dB}$ the results become poorer and poorer to the extent of being useless owing to the extremely high errors. The abrupt degradation of the results is a consequence of the sensitive dependence on initial conditions of chaotic systems. This can clearly be seen in the inset of Fig. 7. There the chaotic attractor for a SNR of $15 \mathrm{~dB}$ is shown. The effect of noise is to blur the spiral so it is increasingly difficult to determine the parameter $r$. This ultimately affects the accuracy of the sensor.

The proposed sensor is also able to measure the attenuation of the fiber. It measures only the total attenuation in the fiber ring, so it can detect changes in this parameter no matter whether they are punctual or distributed. Figure 8 shows the dependence of the maximum radius of the spiral with the attenuation factor of the fiber for a $2400 \mathrm{~m}$ long fiber ring and an attractor formed by 10,000 pulses. Thus, by measuring the maximum radius of the spiral, one can determine the attenuation of the fiber. In fact, this sensor, owing to its longitude, is very sensitive to changes in the attenuation factor of the fiber, and variations as small as $0.01 \mathrm{~dB} / \mathrm{km}$ can easily be detected. How-

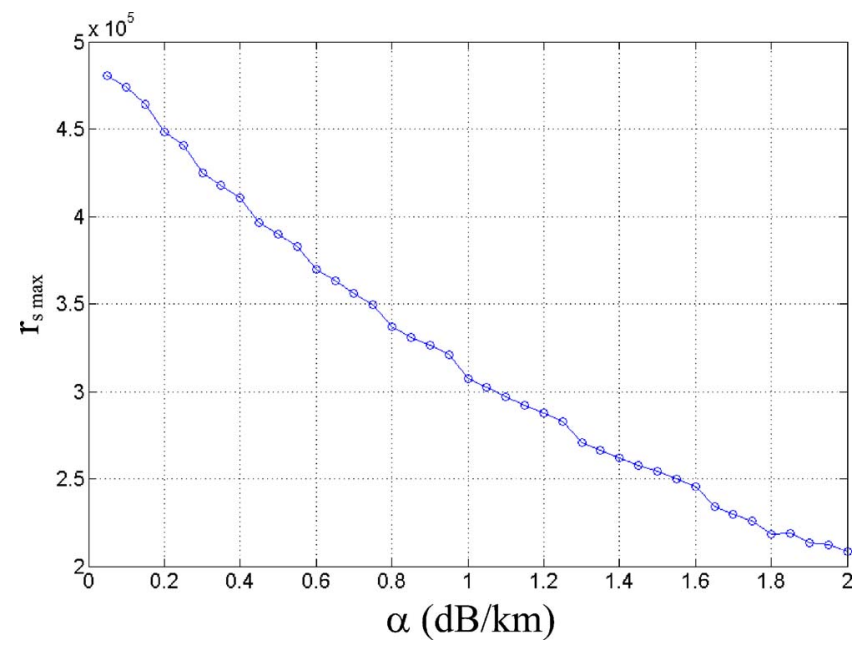

Fig. 8. (Color online) Evolution of the maximum radius of the chaotic attractor with the attenuation factor of the fiber.

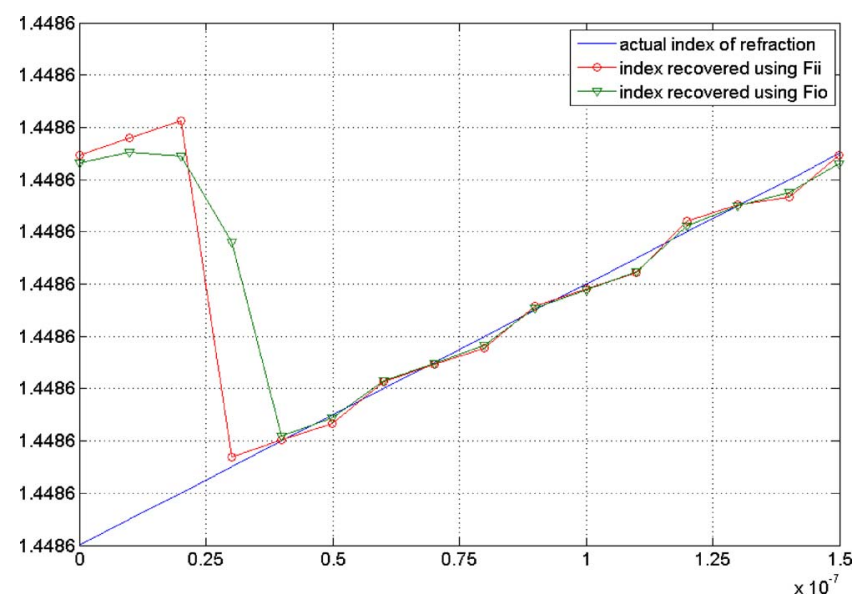

Fig. 9. (Color online) Index of refraction recovered using the information provided by $\phi_{i}$ (dotted curve) and $\phi_{o}$ (curve with triangles), as compared with the actual values (solid curve). ever, obtaining a well-defined attractor is of particular importance in this case, since the measurement relies on a single point.

Finally, the sensor is able to measure yet another parameter: index of refraction. It was shown in Section 3 that the angular parameters of the model $\left(\phi_{i}, \phi_{f}\right.$, and $\left.\phi_{o}\right)$ depend on the index of refraction of the fiber $n_{1}$. With Eqs. (6), this parameter can be retrieved from the angles that define the best fit of the model to the actual attractor. Thus Fig. 9 presents the measurement of the index of refraction from a $1500 \mathrm{~m}$ long fiber resonator recovered from the values of $\phi_{i}$ and $\phi_{o}$. The quality of the data recovered using $\phi_{f}$ is much worse. The reason is that, to obtain a good estimation of $\phi_{f}$, a good definition of the attractor is required owing to the lower probability of points lying in the outer parts of the attractor. Therefore, this parameter has been discarded as a reliable source for the measurement of the index of refraction.

It can be seen in Fig. 9 that the measurements of the index of refraction obtained using either $\phi_{i}$ (dotted curve) or $\phi_{o}$ (curve with triangles) are good. In fact, the mean error is around $1.2 \times 10^{-9}$. This could be enough to measure the changes induced in the fiber by electrical currents by means of the Faraday effect. Even though the results obtained from $\phi_{i}$ and $\phi_{o}$ are similar in this case, in general those coming from $\phi_{0}$ are slightly better. This is because $\phi_{o}$ is a stronger parameter in the sense that it is obtained by fitting many points of the attractor in contrast to $\phi_{i}$, which relies on the measurement of a single point.

\section{CONCLUSIONS}

This paper analyzes the feasibility of using chaos to sense different physical parameters. This will give rise to a new family of fiber sensors: the chaotic sensors. In this paper one of the most simple of these sensors is thoroughly analyzed from the point of view of its usefulness for sensing. The structure of this sensor consists of a fiber-ring resonator optically driven by a train of input pulses. It is seen, by means of multiple simulation results, that the geometrical characteristics of the chaotic attractor of such sensor depend on various physical parameters: fiber length, index of refraction, and attenuation of the fiber are the most important ones. Then, provided the chaotic attractor could be measured, this structure would allow the measurement of all those parameters, becoming thus, in essence, a multiparameter sensor.

However, to recover the measurands from the output data, it is necessary to elaborate a model for the chaotic attractor that allows fitting it. From this fit, the geometrical characteristics of the actual attractor will be determined, and the values of the physical parameters affecting them will be recovered. Such mathematical model is presented in this paper and thoroughly analyzed and discussed.

Finally, the validity of the model is put to test in multiple simulations, leading to determination of the quality of the measurements of the chaotic sensor. Results are promising for all the physical parameters analyzed.

\section{ACKNOWLEDGMENTS}

La Fundación Ramón Areces is gratefully thanked for the economic support given to César Jáuregui through the 
grant ampliación de estudios en al extranjero. The Spanish Ministerio de Educación y Ciencia is also thanked for the support through the TEC2004-05936-C02 project. The work was also partly supported by the Universidad del Pais Vasco-Euskal Herriko Unibertsitatea, Gobierno Vasco-Eusko Jaurlaritza, and the Ministerio de Ciencia y Technologia through the projects GIU05/03, UE05/A25, HEGATEK-05, SENSOFIB/SAIOTEK, and TIC200308361 .

C. Jáuregui, the corresponding author, can be reached by e-mail at cesar.jauregui@gmail.com.

\section{REFERENCES}

1. E. N. Lorenz, "Deterministic nonperiodic flow," J. Atmos. Sci. 20 130-141 (1963).

2. K. Suzuki, Y. Imai, and F. Okumura, "Proposal for temperature and pressure sensing based on periodic chaos synchronization in optical fiber ring resonator systems," in Proceedings of Optical Fibre Sensors Conference Series 16 , K. Hotate, ed. (Institute of Electronics, Information and Communication Engineers, 2003), pp. 204-207.

3. K. Ikeda, H. Daido, and O. Akimoto, "Optical turbulence: chaotic behavior of transmitted light from a ring cavity," Phys. Rev. Lett. 45, 709-712 (1980).

4. J. Gleick, Chaos-Making a New Science (Seix Barral, 1998). 